\title{
The LHCb Trigger System
}

\author{
J. Albrecht ${ }^{\mathrm{a} *}$ \\ On behalf of the LHCb collaboration \\ ${ }^{a}$ Physikalisches Institut, Universität Heidelberg, \\ Philosophenweg 12, 69120 Heidelberg, Germany
}

The Large Hadron Collider beauty experiment $(\mathrm{LHCb})$ is a dedicated B physics experiment at the LHC. The trigger system is of crucial importance to discriminate the interesting B meson decays against the dominant background from inelastic pp-scattering. In this note, an overview of the LHCb trigger system will be given. We put a special emphasis on the software trigger level.

\section{Introduction}

The bbar quark pair production cross section at $\sqrt{s} \approx 14 \mathrm{TeV}$ is approximately $0.5 \mathrm{mb}$. This makes the LHC collider an ideal facility to study B-meson decays. The LHCb experiment is designed to operate with an average luminosity of $2 \cdot 10^{32} \mathrm{~cm}^{-2} \mathrm{~s}^{-1}$ such that events are dominated by single proton-proton interactions. At this luminosity, collisions are expected to take place at a rate of $10 \mathrm{MHz}$. Out of these events, $15 \mathrm{kHz}$ of $b \bar{b}$ pairs are expected to decay fully contained in the spectrometer acceptance. The LHCb physics program is discussed within these proceedings in [1].

The LHCb Trigger [2] is of crucial importance to select amongst the collisions those that are of interest for B-physics studies. The trigger is based on a two level system and exploits the fact that b-flavored hadrons are relatively heavy and long lived.

The first level trigger, called L0, is implemented in hardware. Its main goal is to select particles with high transverse energy and transverse momentum using partial detector information. This trigger level reduces the rate from $10 \mathrm{MHz}$ to an output rate of $1.1 \mathrm{MHz}$. At this rate, the full detector is read out. The second trigger level is a software application running on an Event Fil-

*Presented at BEACH 2008, the Eighth International Conference on Hyperons, Charm and Beauty Hadrons at the University of South Carolina, Columbia, 22 - 28 June 2008. ter Farm which will comprise about $16000 \mathrm{CPU}$ cores. In a first step, the hardware decision is confirmed using partial event reconstruction. Events which pass this confirmation are then fully reconstructed and a complete analysis of interesting B decays is performed. The output rate of the software trigger is $2 \mathrm{kHz}$, at which data is saved to mass storage.

\section{The LHCb Spectrometer}

The $\mathrm{LHCb}$ detector [3] is a single arm spectrometer, whose layout can be seen in Fig. 1, covers an acceptance of 10-300 mrad in X and 10$250 \mathrm{mrad}$ in $\mathrm{Y}$. The $\mathrm{LHCb}$ detector consists of a vertex locator (VELO), a 4 Tm warm dipole magnet, a tracking system (TT, IT, OT), two ring imaging Cherenkov detectors, a calorimeter system and a muon system. We will focus here on the subdetectors which play a major role in the trigger.

The tracking system consists of the Vertex Locator (VELO) around the interaction point and the main tracker after the magnet. The VELO is built of 21 stations with semi-circular silicon sensors measuring the radial and azimuthal coordinates. The sensors can be placed at $8 \mathrm{~mm}$ to the beam line during stable beam conditions or can be retracted during beam injection. The main tracking system consists of silicon sensors in the inner part (IT), a cross shaped area around the beam pipe and drift tubes in the outer part 


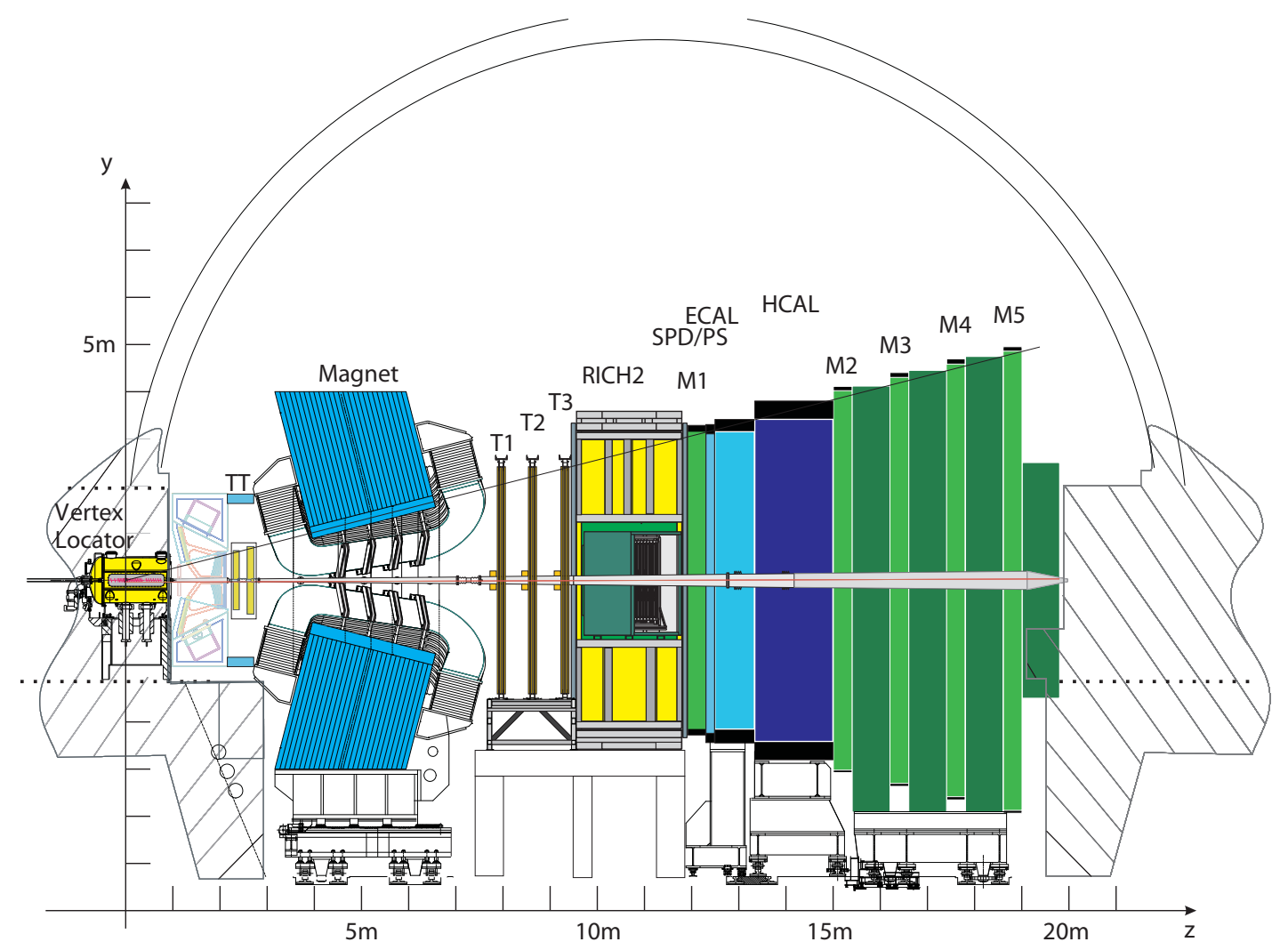

Figure 1. LHCb detector layout, showing the Vertex Locator (VELO) the dipole magnet, the two RICH detectors, the four tracking stations TT, T1-T3 (main tracker), the Scintillating Par Detector (SPD), Preshower (PS), Electromagnetic (ECal) and Hadronic (HCal) calorimeters and the five muon stations M1-M5

(OT). The tracking system is able to measure the track impact parameter with a resolution down to $14 \mu \mathrm{m}$ and the track momentum with a resolution of $0.4 \%$.

The calorimeter system [4] consists of a Scintillating Pad Detector (SPD), a Pre-Shower Detector (PS) followed by electromagnetic (ECal) and hadronic (HCal) calorimeters. The Shashlik type calorimeter elements are based on scintillating tiles, read out to photomultipliers via wavelength-shifting fibers. The scintillators are interleaved with lead for the ECal and with iron tiles for the HCal.
The ECal depth accounts for 25 electromagnetic interaction lengths $\left(\mathrm{X}_{0}\right)$ and 1.1 hadronic interaction lengths $(\lambda)$, its energy resolution is $\sigma(\mathrm{E}) / \mathrm{E}=10 \% / \sqrt{E} \oplus 1.5 \%$ (with $\mathrm{E}$ in $\mathrm{GeV}$ ). Cell dimensions are varying in the range of $4 \times 4 \mathrm{~cm}^{2}$ to $12 \times 12 \mathrm{~cm}^{2}$.

The HCal depth accounts for $5.6 \lambda$, its energy resolution is $\sigma(\mathrm{E}) / \mathrm{E}=80 \% / \sqrt{E} \oplus 10 \%$ (with $\mathrm{E}$ in $\mathrm{GeV}$ ). The HCal cell dimensions vary from $13 \times 13 \mathrm{~cm}^{2}$ to $26 \times 26 \mathrm{~cm}^{2}$.

The muon system consists of five stations. The first station (M1) is located before the calorimeter system to improve the momentum 
measurement, whereas the other stations (M2M5) are located after the calorimeters. The muon stations are equipped with multi-wire proportional chambers, and are separated by iron filters. For the innermost region of the first muon station, where the particle density will be higher, triple-GEM detectors are used.

\section{Hardware Trigger: L0}

The LHCb detector will see bunch crossings with at least one visible interaction at a rate of $10 \mathrm{MHz}$. The purpose of the L0 trigger is to reduce the rate to $1.1 \mathrm{MHz}$ with which the entire detector can be read out. The L0 trigger reconstructs and selects particles with high transverse momentum in the muon chambers or with high transverse energy in the calorimeter system.

\subsection{The L0 Muon Trigger}

The L0 muon trigger looks for muon tracks with a high transverse momentum, $\mathrm{p}_{T}$. The track finding searches for hits on a straight line through the five stations, giving a high purity muon identification. Due to the high multiple scattering between the stations, only hits from the first two stations together with a pointing constraint to the interaction point are used to determine the $\mathrm{p}_{T}$ of the muon candidate. A momentum precision of $\sigma_{p} / p \approx 20 \%$ is achieved by the Level-0 processor, assuming all muons originate from the primary vertex. The two muon tracks with the highest $\mathrm{p}_{T}$ are selected for each quadrant of the muon stations. This results in up to eight muon candidates which are selected by the L0 muon trigger.

\subsection{The L0 Calorimeter Trigger}

The Level-0 calorimeter trigger reconstructs and selects particles with a high transverse energy deposit in the calorimeters. It provides candidates for hadrons, electrons, photons and neutral pions. The transverse energy is measured in the ECal and HCal. As the expected showers of $\mathrm{e}^{ \pm}, \gamma$ and single hadrons are relatively narrow, a zone of 2 by 2 cells is used, large enough to contain most of the energy and small enough to avoid overlaps between different particles. The properties of the showers together with the SPD and PS detector information provide a first parti- cle hypothesis. For each type, only the candidate the highest $\mathrm{E}_{T}$ is kept. The SPD detector before the ECal is also used to measure the charged track multiplicity.

\subsection{The L0 Pile-Up System}

The pile-up veto system is a component of the VELO and is made of two silicon planes, positioned upstream (with respect to the spectrometer) of the nominal interaction point. The pile-up veto provides a rejection of events with multiple proton-proton collisions through the reconstruction of all primary vertices in the event. Events with two interactions are identified with an efficiency of $60 \%$ and a purity of about $95 \%$.

\subsection{L0 Bandwidth Share and Performance}

The thresholds for the L0 trigger channels are summarized in Tab. 1. A L0 hadron trigger is issued if the transverse energy of a cluster of hadronic type is above $3.5 \mathrm{GeV}$. Additionally, events with multiple interactions or high track multiplicity are vetoed. A L0 muon trigger is issued if the muon candidate with the highest $\mathrm{p}_{T}$ is above the threshold $(1.3 \mathrm{GeV})$ or if the sum of the two highest $\mathrm{p}_{T}$ candidates is above $1.5 \mathrm{GeV}$.

These thresholds lead to a rate of $\sim 700 \mathrm{kHz}$ for the L0 hadron trigger, $\sim 200 \mathrm{kHz}$ for the muon trigger and $\sim 200 \mathrm{kHz}$ for the electromagnetic trigger. The total output rate of the L0 trigger is $1.1 \mathrm{MHz}$, about $10 \%$ of the events are triggered by more than one L0 trigger type.

The performance of the Level-0 trigger is measured on events that pass the offline B-selection algorithms used for physics analyses. The L0 trigger efficiency is approximately $50 \%$ for hadronic channels (i.e. $\mathrm{B}_{s} \rightarrow \mathrm{D}_{s} \pi$ ), $90 \%$ for muonic channels (i.e. $\mathrm{B}_{s} \rightarrow \mathrm{J} / \psi(\mu \mu) \phi$ ) and $70 \%$ for electromagnetic channels (i.e. $\mathrm{B} \rightarrow \mathrm{K}^{*} \gamma$ ).

The total latency of the L0 trigger is $4 \mu \mathrm{s}$, while the latency of the reconstruction algorithms in the calorimeter and muon detectors is $1 \mu \mathrm{s}$.

\section{Software Trigger: HLT}

The High Level Trigger (HLT) is the second and last trigger level of $\mathrm{LHCb}$. It is a $\mathrm{C}++$ application running on Level-0 accepted events at a rate of $1.1 \mathrm{MHz}$. At this rate, the full detector in- 
Table 1

The total output of the L0 trigger is $1.1 \mathrm{MHz}$. It is composed as a logical OR of the hadronic, the muonic and the electromagnetic trigger decision. About $10 \%$ of the events are triggered by more than one L0 trigger.

\begin{tabular}{llccccc}
\hline & hadronic & \multicolumn{2}{c}{ muonic } & \multicolumn{3}{c}{ electromagnetic } \\
& & $\mu$ & di- $\mu$ & $e^{ \pm}$ & $\gamma$ & $\pi^{0}$ \\
\hline threshold & & & & & \\
pt $/$ Et $(\mathrm{GeV})$ & 3.5 & 1.3 & $\Sigma>1.5$ & 2.6 & 2.3 & $4-4.5$ \\
\hline rate $(\mathrm{kHz})$ & 700 & \multicolumn{2}{c}{200} & & 200 & \\
\hline
\end{tabular}

formation is read out into the Event Filter Farm. The connection between the detector front-end buffers and the EFF is provided by a high performance network with a data throughput of about $50 \mathrm{GBytes} / \mathrm{s}$.

The HLT is divided in two levels (see scheme Fig. 2): The HLT first level reduces the rate from the $1.1 \mathrm{MHz}$ output of the L0 trigger to a few tens of $\mathrm{kHz}$. It applies different sequences of algorithms (called alleys) dependent on the decision issued by the Level-0 trigger. It uses only a part of the full detector information available to decrease the required processing power. The strategy is to confirm the Level-0 candidates by adding information from either the VELO or the main tracker and applying cuts on the transverse momentum $\left(\mathrm{p}_{T}\right)$ and the impact parameter (IP) with respect to the primary vertex. For some alleys, additional particles apart from the Level-0 candidate are searched for to give a better discrimination between B-decay products and background.

On these events, the HLT second level is executed. It first performs a full pattern recognition on the remaining events. On the fully reconstructed events, a combination of inclusive selection of specific resonances (such as $\mathrm{J} / \psi$ or $\mathrm{D}^{*}$ ) and exclusive reconstruction of B-hadron final states is performed. The final trigger decision is an OR between the inclusive and exclusive selections.

Both the tracking and the vertexing achieve a precision comparable to what is later obtained offline. As an example, a momentum precision close to $\sigma_{p} / \mathrm{p} \approx 1 \%$ is obtained for tracks traversing the whole tracking system; for comparison the offline precision is $\sigma_{p} / \mathrm{p} \approx 0.4 \%$ for the same trackers.

\subsection{HLT First Level}

The HLT first level consists of several parallel sequences of algorithms, called trigger alleys. Dependent on the type of the Level-0 trigger decision, a different alley is executed. The alleys enrich the $\mathrm{B}$ content of the events by adding information from further subdetectors.

While the alleys are operating independently, care has been taken to avoid having to reconstruct the same track or vertex twice to avoid wasting precious CPU power. For the same reason, each alley is interrupted as soon as one of its filtering or reconstruction steps outputs no suitable candidate.

\subsubsection{L0 Confirmation with Trackers}

The first step in each alley is to confirm the Level-0 candidate with an additional subdetector. This can be the VELO and / or the main tracker. The following algorithms are used to confirm Level-0 objects:

Level-0 + Main Tracker: To confirm of discard the track assumption for the Level-0 object, a corresponding track candidate is searched for in the main tracker. The position and energy of the L0 candidates $\left(\mathrm{h}, \mu, \mathrm{e}^{ \pm}, \gamma\right)$, together with the assumption to originate from the interaction region, define the whole trajectory of the candidate in the detector. In the case that the L0 candidate is a calorimeter cluster, two trajectories for the two charge assumptions are defined. The hits in the tracker are only decoded in a parameterized region around the calculated trajectory. Pattern recognition is only done on these hits. To further improve the track finding time, the tracks are required to match the L0 object in position 


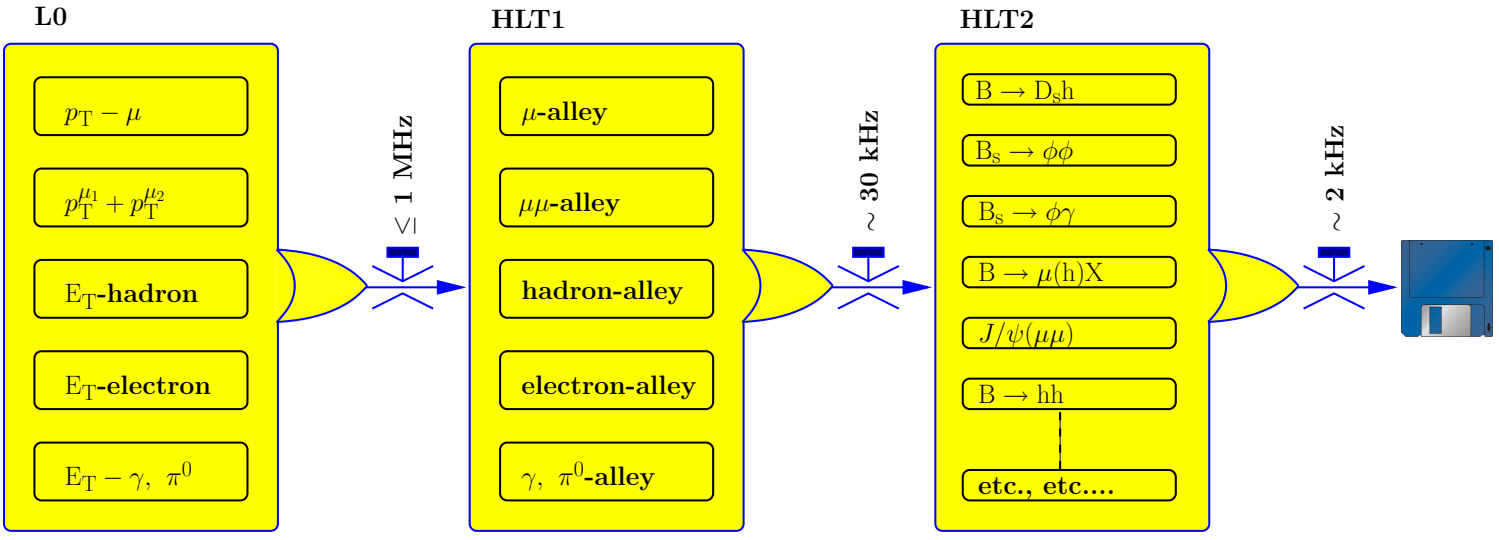

Figure 2. Overview of the LHCb trigger scheme. Only a subset of the HLT first level schemes are applied dependent on the L0 decision. The HLT second level selections are run for every event accepted by the previous level, independent of the trigger type of the Level-0 and the HLT first level.

and direction already in each step of the track finding. By this, tracks which do not match to the L0 candidate can be removed at the earliest possible stage.

Additionally to the confirmation of the track assumption, the $\mathrm{p}_{T}$ resolution is significantly improved by this algorithm: The $\mathrm{E}_{T}$ resolution for hadronic L0 candidates is $\approx 30 \%$ (for muons $\approx 25 \%$ ). The track confirmation in the main tracker improves this to about $3 \%$, see Fig. 3 .

Level-0 + VELO: Track segments in the VELO are reconstructed in two stages. First the information from the R-sensors is used to reconstruct 2-dimensional tracks. The 2-dimensional tracks are matched to the L0 candidates and only tracks pointing to the L0 object are used to reconstruct full 3-dimensional tracks using the phisensor information. These tracks are used to calculate a 3 -dimensional matching $\chi^{2}$ with the L0 object. In addition, the 2-dimensional tracks are used to reconstruct the primary vertices in the event.

VELO + Main Tracker: The confirmed VELO tracks together with the matched L0 ob- ject define a trajectory in the whole detector. Using this trajectory, track search in the main tracker is done completely analogously to the $L O$ + main tracker algorithm described above.

Main Tracker + VELO: Candidates that have been confirmed in the main tracker are matched to VELO tracks using an algorithm completely analogously to the $L O+V E L O$ case described above. However, the precision of a track extrapolated to the VELO is much higher than the one of a L0 object, leading to tighter cuts on the matching $\chi^{2}$.

\subsubsection{Extra tracks}

In some alleys, the confirmation of the L0 object is complemented with the search of an extra track to provide sufficient rate reduction.

Dimuons: Due to bandwidth restrictions, the L0 trigger has to select the L0 objects with the highest $\mathrm{p}_{T}$, see above. In case of one L0 muon candidate which is confirmed in the trackers, a search for a second muon signature is done in M2M5. This additional candidate is then confirmed with the trackers as described above. The found dimuon system should form a good vertex.

Di-hadrons: When a L0 hadron candidate is confirmed, a search is performed in the VELO 


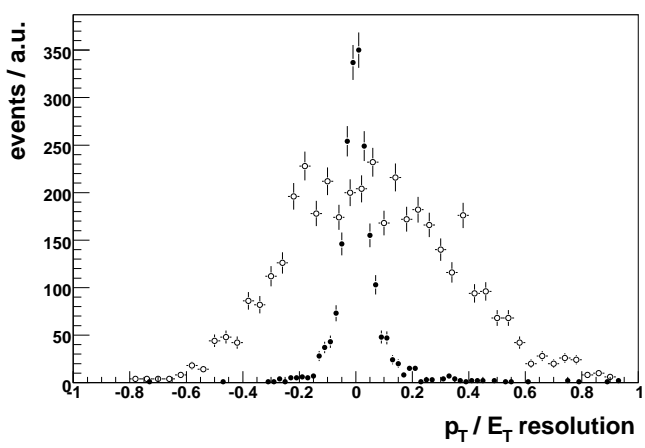

Figure 3. The closed points show the $\mathrm{p}_{T}$ resolution for tracks reconstructed in the HLT first level on simulated events, $\sigma\left(\mathrm{p}_{T}\right) / \mathrm{p}_{T} \approx 3 \%$. The open points show the $\mathrm{E}_{T}$ resolution for hadronic candidates, $\sigma\left(\mathrm{E}_{T}\right) / \mathrm{E}_{T} \approx 30 \%$.

for tracks forming a good vertex with it. If such a track is found, it is then confirmed in the main tracker.

Muon+track: In a similar way, when a L0 muon candidate is confirmed, a search is performed at the VELO for tracks forming a good vertex with it. If it is found, it is then confirmed in the main tracker. In contrast to the dimuon case, the second track is not required to have hits in the muon stations.

\subsubsection{Decision}

If the L0 candidates are confirmed successfully, the trigger decision is then taken, based on the $\mathrm{p}_{T}$ and impact parameter of the confirmed candidates. For alleys requiring an extra track, the decision is based also on the $\mathrm{p}_{T}$ and IP of the extra track, as well as on the vertex quality (distance of closest approach of the two tracks, DOCA).

In case of muons, a significant fraction of dimuons is triggered without a cut on the impact parameter. The dimuon system is required to either have an IP $>150 \mu \mathrm{m}$ and an invariant mass of larger than $500 \mathrm{MeV}$ or an higher invariant mass $\left(\mathrm{m}_{\mu \mu}>2500 \mathrm{MeV}\right)$ and no IP require- ment. This allows an analysis of lifetime unbiased B-decays, for example $\mathrm{B}_{S} \rightarrow \mathrm{J} / \psi \phi$.

Each alley produces summary information which is written to storage for accepted events. This summary contains the information of all tracks and vertexes which triggered the event.

\subsubsection{Example of HLT Alley: Hadron Alley via Tracker}

The input of the HLT first level hadron alley will be $700 \mathrm{kHz}$. At this high rate, a fast rate reduction is essential. As discussed above, there are two ways to confirm a Level-0 candidate: With tracks from the VELO or with tracks from the main tracker. Both confirmation strategies are developed in parallel and the decision which algorithms to use will be taken dependent on the performance in terms of CPU time and efficiency as measured on data. As an example, the hadron alley with L0 confirmation with the main tracker will be presented here.

The input from the L0 hadron contains on average 1.3 candidates per event. The L0 calorimeter clusters are then confirmed with tracks from the main tracker, as discussed above. The reconstructed track is required to have a $\mathrm{p}_{T}$ larger than $2.5 \mathrm{GeV}$. This reduces the rate to $350 \mathrm{kHz}$, with on average 1.2 found tracks per hadron candidate. These tracks are then matched to the VELO and an impact parameter larger than $100 \mu \mathrm{m}$ is required. The requirement that a track needs to have both a high IP and large $\mathrm{p}_{T}$ reduces the rate to $30 \mathrm{kHz}$ with 1.0 found tracks per hadron candidate.

Since this rate is still too large for the HLT2 stage, a further reduction is obtained by requiring an extra VELO-track with a distance of closest approach to the confirmed Level-0 track of less than $0.2 \mathrm{~mm}$, and a $\mathrm{p}_{T}$ of at least $1 \mathrm{GeV}$. This reduces the rate to $11 \mathrm{kHz}$ with 2.1 candidate secondary vertexes reconstructed per L0 hadron candidate.

This is the output of the HLT1 hadron alley, the other HLT1 alleys employ similar strategies.

\subsection{HLT Second Level}

After the HLT first level, the events are fully reconstructed. This happens at a rate of a few tens 
of $\mathrm{kHz}$, depending on the CPU performance of the reconstruction software. Tracks in the VELO are used as seeds for the rest of the tracking. The HLT2 tracks differ from the offline reconstructed tracks in not having been fitted with a Kalman filter to obtain the full covariance matrix since this is too CPU intense.

A set of tracks is selected with very loose cuts on their momentum and impact parameter. These tracks are used to form composite particles, like $\mathrm{K}^{*} \rightarrow \mathrm{K}^{+} \pi^{-}, \phi \rightarrow K^{+} K^{-}, \mathrm{D}^{0} \rightarrow \mathrm{hh}$, $\mathrm{D}_{s} \rightarrow K^{+} K^{-} \pi^{-}$and $\mathrm{J} / \psi \rightarrow \mu^{+} \mu^{-}$, which are subsequently used for all selections to avoid duplication in the creation of final states.

Two types of final selections are applied: inclusive and exclusive. Inclusive selections aim to collect decays of resonances which are likely to have been produced in $\mathrm{B}$ decays $\left(\mathrm{D}^{*}, \mathrm{~J} / \psi\right.$, etc.) and which are useful for lifetime unbiased physics analyses and calibration. Exclusive selections are specifically designed to provide the highest possible efficiency for fully reconstructed $\mathrm{B}$ decays of interest.

\subsubsection{Inclusive Selections}

The inclusive trigger selections have three streams of data: a $\mathrm{D}^{*}$ line, a line with di-muons and a line with events containing single muons:

The $\mathbf{D}^{*}$ inclusive trigger selects the clean decay $\mathrm{D}^{*+} \rightarrow \mathrm{D}\left(\mathrm{K}^{+} \pi^{-}\right) \pi^{+}$. This sample can be used, e.g. to calibrate the particle identification performance of the RICH detectors. It also enhances the potential of $\mathrm{LHCb}$ to study $\mathrm{CP}$ violation in charm decays. It is foreseen that this hadronic selection triggers at a rate of $300 \mathrm{~Hz}$.

The di-muon inclusive selection reconstructs muon pairs with a high invariant mass or with a high impact parameter and a lower invariant mass requirement. For example, $\mathrm{J} / \psi$ 's from $\mathrm{B}_{S} \rightarrow$ $\mathrm{J} / \psi \phi$ are reconstructed in the HLT with an invariant mass resolution of below $20 \mathrm{MeV}$ [5]. Avoiding to cut on the impact parameter, this trigger line allows the selection of di-muons with no bias on the lifetime of the parent B meson. The dimuon trigger line will contribute $600 \mathrm{~Hz}$ to the final trigger output.

The single muon inclusive trigger provides a high purity $\mathrm{B} \rightarrow \mu \mathrm{X}$ sample that can be used to understand the interplay between trigger and tagging. It provides a very large sample of events triggered independently of the B-decay "opposite" to the muon. The single muon inclusive trigger will contribute $900 \mathrm{~Hz}$ to the final trigger output.

\subsubsection{Exclusive Selections}

The exclusive trigger selects specific B decay channels. Approximately 100 core physics channels including control channels are selected. Full reconstruction and analysis is performed using all available information including mass and vertex quality. As the resolution of the online tracking is worse than the offline resolution, wide cuts are used. As an example, $\mathrm{B} \rightarrow \pi \pi$ events are selected requiring two tracks which make a good vertex and have an invariant mass in a wide range of $600 \mathrm{MeV}$ around the B mass. This is to be compared to the offline selection which applies a masss window cut of $50 \mathrm{MeV}$. In addition, the $B$ candidate should have a significant impact parameter. That results in an efficiency to select $\mathrm{B} \rightarrow \pi \pi$ events of $88 \%$. The exclusive selections trigger events with a rate of $200 \mathrm{~Hz}$.

The main background for the events selected by the exclusive selections are the $15 \mathrm{kHz}$ of other Bhadrons which decay in the spectrometer acceptance.

\section{Trigger Performance}

The efficiency of the LHCb trigger system is summarized in Tab. 2 for hadronic, electromagnetic and muonic B-decays. As before, the trigger efficiency is measured on events that pass the offline physics analysis selection cuts.

A prototype of the full HLT first and second level trigger has been tested and profiled. The Event Filter Farm processed 100 events/second per CPU. This is well within the time budged for running the HLT with an input rate of $1.1 \mathrm{MHz}$.

\section{Outlook}

The current status and expected performance of the LHCb trigger system has been presented. The strategy for the Level-0 trigger, which is based on custom built trigger processors, is well 
Table 2

Summary of Trigger performance. The efficiency of the channel $\mathrm{B}_{s} \rightarrow \mathrm{J} / \psi \phi$ is given for the lifetime unbiased trigger selection. If a lifetime cut is applied in the trigger, the muonic HLT efficiency increases to above $90 \%$.

\begin{tabular}{lllll}
\hline Type & example & L0 & HLT & total \\
\hline hadronic & $\mathrm{B} \rightarrow \mathrm{h} \mathrm{h}$ & $50 \%$ & $80 \%$ & $40 \%$ \\
$\mathrm{e} / \mathrm{m}$ & $\mathrm{B} \rightarrow \mathrm{K}^{*} \gamma$ & $70 \%$ & $60 \%$ & $40 \%$ \\
muonic & $\mathrm{B}_{s} \rightarrow \mathrm{J} / \Psi(\mu \mu) \phi$ & $90 \%$ & $80 \%$ & $70 \%$ \\
\hline
\end{tabular}

defined. It shows good performance for the $\mathrm{LHCb}$ physics program. The production and installation of the L0 trigger is completed, commissioning is in its final stage.

The LHCb software trigger that will run on an 16000 CPU Event Filter Farm is also well established and works within the time budget. The HLT will efficiently select B meson decays relevant for physics studies and inclusive samples to be used for calibration purposes and to study systematic effects. The final event rate to permanent storage is $2 \mathrm{kHz}$.

For the 2008 physics run 1600 cores of the EFF have been installed and commisioned, and the system will be completed early 2009 .

\section{REFERENCES}

1. S. T'Jampens, The LHCb Physics Program, this proceedings

2. The LHCb Collaboration, LHCb Trigger System Technical Design Report, CERN/LHCC 03-031.

3. The LHCb Collaboration, The LHCb Detector at the LHC, 2008 JINST 3 S08005

4. The LHCb Collaboration, $\mathrm{LHCb}$ Calorimeters Technical Design Report, CERN/LHCC 00-036.

5. L. Fernandez, P.Koppenburg, Exclusive HLT Performance, LHCb note 2005-047 